\title{
Dam Automation using IoT
}

\author{
Dr. Nagesha Shivappa \\ Associate Professor, Dept. of E\&IE. \\ JSS Academy of Technical Education. \\ Bengaluru, India.
}

\author{
Aishwarya S Rao, Aishwarya T, \\ Jahnavi S Athreya, Mandakini H. \\ Dept. of Electronics and Instrumentation Engg. \\ JSS Academy of Technical Education. \\ Bengaluru, India.
}

\begin{abstract}
Dams play a very important role to hold and conserve water for optimal usage based on seasonal needs. Water Management plays a very important role in mitigating the current issues of water distribution and utilization. As there are lot of hazards related with the existence of the dams, it has become a necessity to develop a proper monitoring system regarding the opening of the dam gate to retain a safe water level in dams. Exploring usage of IoT for improving the safe utilization of dams, water flow and prevention of dam gate corrosion. This paper intends to use microcontroller for monitoring and controlling the water distribution management by usage of various sensors, control valves, automatically \& proactively manage outflow during crisis by using statistical data of the environment.
\end{abstract}

Keywords: - Control valves, corrosion, IoT, Raspberry Pi.

\section{INTRODUCTION}

Nowadays Water management is an issue of growing concern, as there is limited availability of consumable water. Owing to unforeseen weather conditions such as heavy rainfall, sudden change of tides and other natural forces leads to natural calamities which causes problems like increase in mortality rate, contamination of consumable water, agricultural problems which may negatively impact the economy of the country. Construction of dams provides water bodies for future, protect the available water from pollution, prevents disputes and over exploitation. Therefore, dams play significant role in water management.

Dams play a prominent source of water supply to the urban networks. Apart from this, dams and reservoirs play an important role in agricultural system. Even today we have been using traditional methods for managing and monitoring the dam gates and measuring the level of water and other parameters. Many factors contribute towards the failure of a dam, the most common being Overtopping which is caused due to heavy floods. This condition needs constant monitoring to avoid catastrophes such as Dam Failure, which is not To overcome the Dam Failure and for the continuous monitoring of Dam Health, the enabling technology of Internet of Things (IoT) is used. Through this the authorities have continuous availability of data about the dam health and they can take appropriate decisions.

Many systems were available for monitoring of dams. Few notable ones related to our research are

[1] are paying attention towards the development of autonomous dam gates system using level sensors and motor controller. Although the dam gates are automatically controlled using DC motor but no information is provided regarding the water level.
[2] have developed a system where the controlling of dam gates is done through the data collected with the help water level sensors deployed near the dam area. This idea will help us to efficiently control the dams all over the country by reducing the manpower for maintenance of the dam. A set of sensors are used to gather information regarding the level of water and then sends instructions back in order to control the dam.

[3] have proposed a system which has the ability to notify the present status of the dam and also alerts when the dam conditions have changed from the normal conditions.

[4] have proposed a concept where the system checks for cracks in walls, increased levels of turbidity in the downstream, which could be a manifestation dam, pipe detoriations and corrosion on the gates of the dam. This obtained information can be displayed in an android application for further decision making by the authorities.

[5], are paying attention Wireless Sensor Network, Android application and ZigBee technology for early flood warning. The sensor network will send details about the water level to the server through ZigBee communication. $\mathrm{ZigBee}$ is connected to dam and main server. The concept also implements the safe zone notification. User can fetch safe zone live mapping with without internet

\section{PROPOSED METHODOLOGY}

The system utilizes different types of sensors to monitor the dam parameters. The system can be divided in to two parts:

\section{A. Automatic Dam Gate Control}

The developed system controls the dam gate automatically by deploying the water level sensors at various stages of the reservoir. Once the dam gate is open the outflow of the water is calculated by employing flow sensor which leads to effective management and utilization of water. Turbidity of water is effectively measured by deploying the turbidity sensor which in-turn prevents the sedimentation caused in the dams.

\section{B. Corrosion Detection}

The corrosion of dam gates is measured by connecting anode and cathode externally and current generated is measured. Since the dam gates are constructed using metal, the ion sensitive element or ion meters are placed on the metallic objects. Hence corrosion of the dam gates is monitored using these methods and the gathered information is conveyed. Based on this information the counter measure of epoxy coating can be done to maintain the good health of the dam gates. 


\section{Flow chart}

The output of the sensors is constantly monitored and updated on the server and displayed on web page. Analysis can be done by using stored data and the system is programmed in such a way that the dam gates are automatically opened whenever the parameter exceeds the threshold value. The data collected can be monitored through a server page. This is shown in "Fig. 1"

\section{SYSTEM DESIGN}

The hardware requirements of the proposed system are as follows:

\section{A. Flow Sensor:}

For the measurement of outflow of the water from dam, flow sensor is employed. It consists of plastic valve body, rotor and hall sensor. The rotor rolls when water flows through it and changes its speed for different rate of flow. The output is the corresponding value of pulse given by hall effect sensor. The flow can be calculated by respective formula.

\section{B. Turbidity Sensor:}

The turbidity of the water is measured by the turbidity sensor by using the principle of optics. It uses an infrared tube by which transmittance and scattering rate of the water is calculated. The output is calculated by corresponding voltage value and it is inversely proportional to the turbidity.

Fig. 1 Flow chart of the proposed system

\section{Raspberry pi:}

The data from the sensors are analyzed using raspberry pi by which necessary actions can be taken. The raspberry pi used here has inbuilt Wi-Fi module due to which cloud application are easier to access. The power supply used is of 5 volts and 2.5 amperes DC which makes it as low power consumption device.

\section{Water Level Sensor:}

The level sensor we are using is known as a Conductive sensor. The liquid which can conduct electricity can be used in this sensor. When a conductive liquid reaches some point, it will come into contact with both longer and shorter electrodes, and thus completing a circuit and activating an internal switch.

\section{E. Corrosion Sensor:}

Corrosion, as an invisible threat, is a potential problem that often remains unnoticed. As the dam gates are always in contact with water on one side and wind on the other

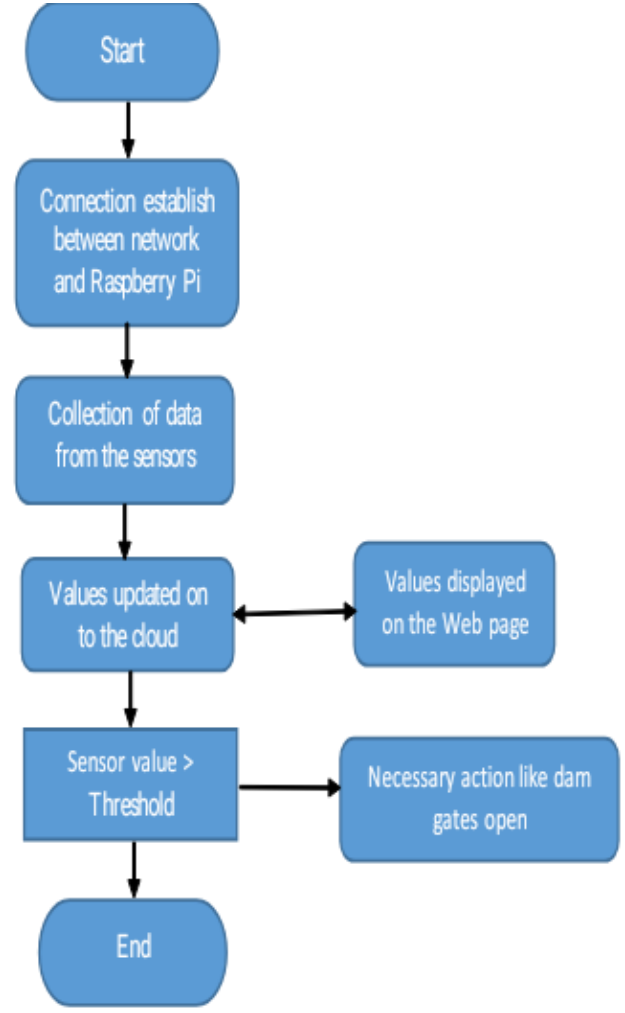

monitoring corrosion data in real-time is a key factor. Access to real-time monitoring of the impact of corrosion provides users with a way to plan and prepare for critical moments in the dam gate's lifetime.

The software requirements are as follows:

\section{F. Python:}

Python 3.0 is used to program the Raspberry Pi. The values of the sensors are updated to the cloud using in built Wi-Fi of the raspberry pi module. 


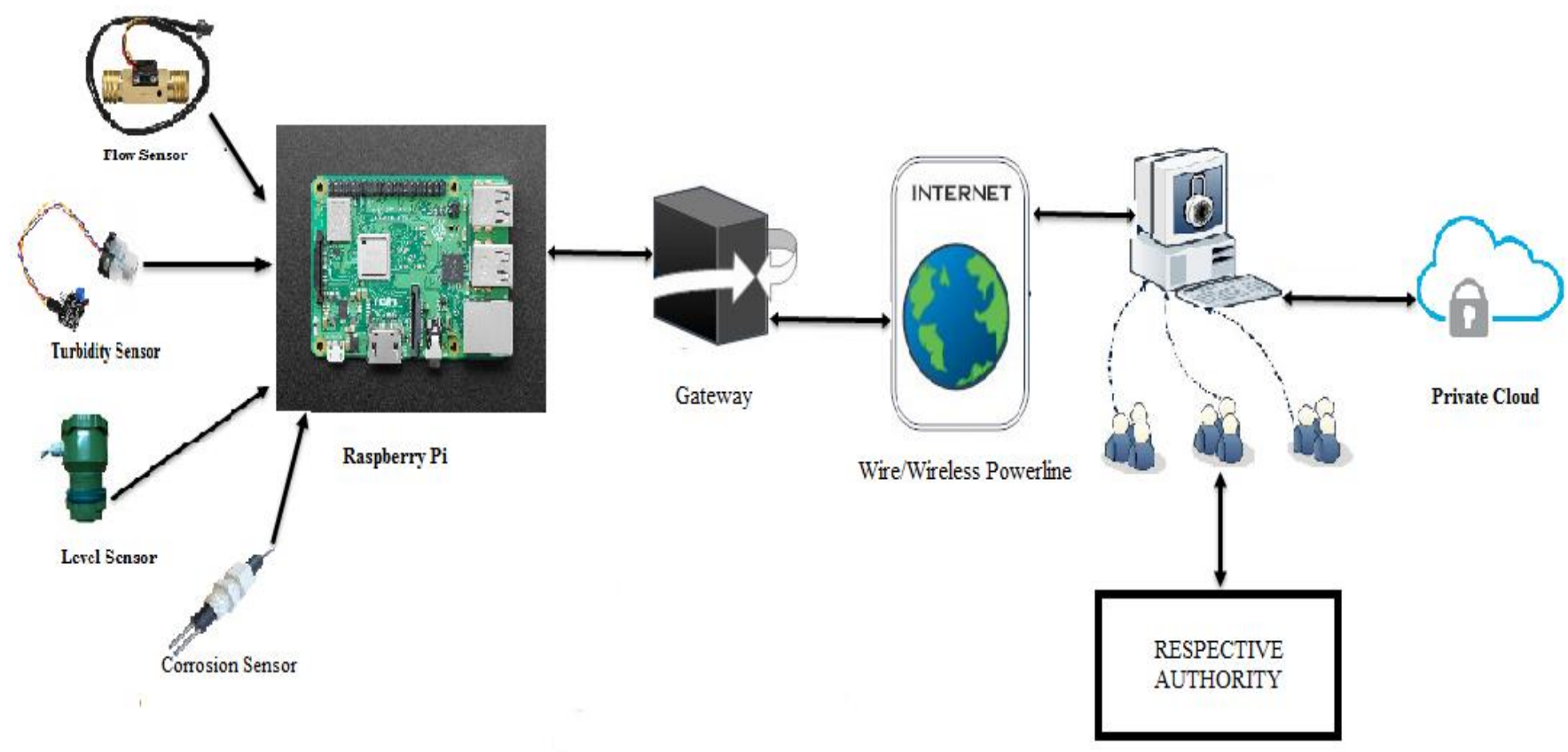

Fig. 2 Architecture of Dam Automation System

\section{G. ThingSpeak:}

It is used to monitor the parameters of the dam. It is an open IoT platform for monitoring your data online. In ThingSpeak channel the data can be set either private or public according to your choice. ThingSpeak can update readings within 15 seconds.

\section{RESULTS}

The prototype of dam monitoring system is developed. First the entire system is switched on and a link is developed between the network and the raspberry pi. The sensors take reading of the respective parameters which are placed on different positions of the dam.

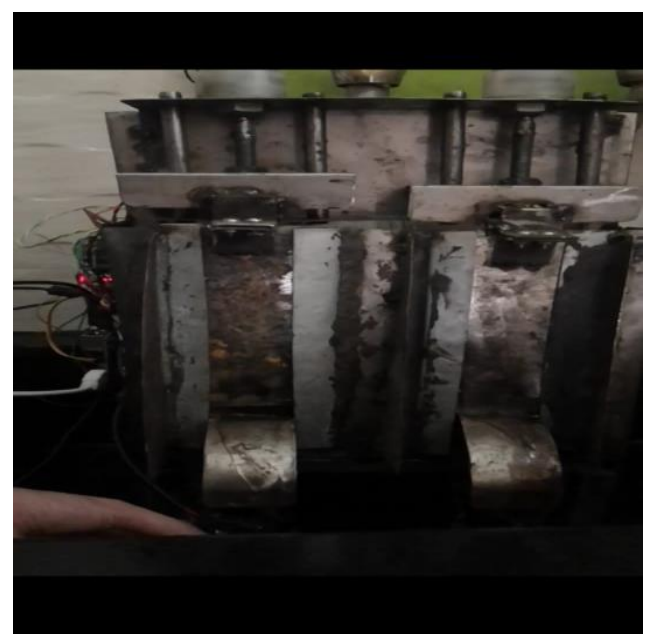

Fig.3 Automatic dam gates open

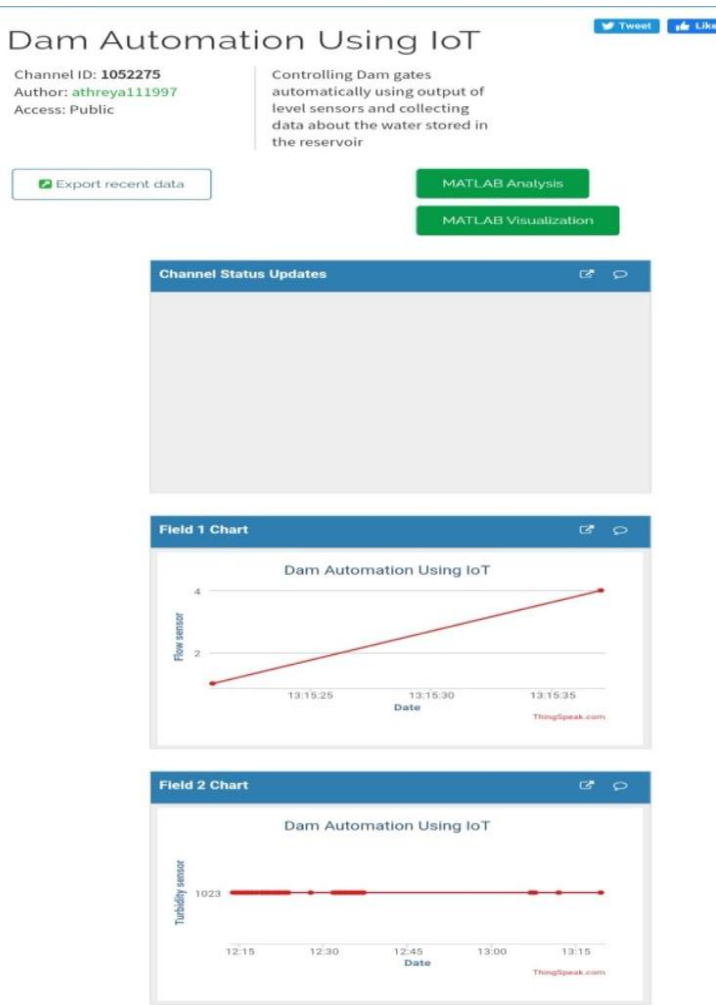

Fig. 4 ThingSpeak Updates

Water flow sensors are placed at the dam gate exit and the amount of water flowing out is displayed on the command window. Turbidity sensor is placed inside the dam and monitors the suspended particles. Metal corrosion sensor is also placed inside the dam and it indicated a message when it come across any metals. All the above data is sent via cloud to ThingSpeak and the data can be viewed by the authority. 


\section{CONCLUSION}

The aforementioned design of the system helps to properly monitor the opening of dam gates. IoT has increased the quality and productivity of dam management system and also conferred to the public issues. To automatically and proactively manage the outflow of dam during crisis by using statistical data. Since the system is using IoT technology, human intervention and time delay is minimized.

\section{REFERENCES}

[1] S.M. Saifur Rahman Faisal, Iftekhar Uddin Ahmed, Humayun Rashid, Remon Das, Md. Mobarak Karim, S M Taslim Reza "Design and Development of an Autonomous Floodgate using Arduino Uno and Motor Driver Controller" Proceedings of the 2017 4th International Conference on Advances in Electrical Engineering 2830 September, 2017, Dhaka, Bangladesh.

[2] Sai Sreekar Siddula, P.C. Jain, Madhur Deo Upadhayay "Real Time Monitoring and Controlling of Water Level in Dams using IoT" 201*IEEE 8th International Advance Computing Conference (IACC).

[3] V. Sathya, Kshitij Arun, Harshitaa Mahajan, Amit Kumar Singh "Automate the Functioning of Dams Using IoT" 2019 3rd International Conference on Computing Methodologies and Communication (ICCMC).

[4] Mrunalini Bajare, Shraddha Kawade, Manali Kamble, Apurva Deshpande, Jayant D. Bokefode "Preventive Maintenance System for Dam Using IOT and Cloud" 2018 Second International Conference on Inventive Communication and Computational Technologies (ICICCT).

[5] Balaji.V, Akshaya.A, Jayashree.N, Karthika.T "Design of ZigBee based Wireless Sensor Network for early Flood Monitoring and Warning system" 2017 IEEE International Conference on Technological Innovations in ICT for Agriculture and Rural Development (TIAR 2017).

[6] Aishwarya S Rao, Aishwarya T, Jahnavi S Athreya, Mandakini H, Nagesha Shivappa, "A Survey Paper on Dam management” IRJET volume 6 , Issue 11 